\title{
Chemotherapy treatment: Colorectal cancer patients' perception
}

\author{
Sonia Betzabeth Ticona-Benavente, Ana Lucia Siqueira Costa \\ Nursing School, Universidade de São Paulo, São Paulo, Brazil
}

Received: November 9, 2017

Accepted: December 26, 2017 Online Published: January 17, 2018

DOI: $10.5430 /$ jnep.v8n6p85

URL: https://doi.org/10.5430/jnep.v8n6p85

\begin{abstract}
Background and objective: The chemotherapy cause greater impact in the physical and emotional sphere and in the experience of the symptoms. Thus, knowing the perception of these patients, is necessary to obtain subsidies that may direct the implementation of effective interventions for the management of adverse events and the support offering regarding the experience of the disease and treatment, with better impact on the quality of life. The objective of this study was to explore the perception of colorectal cancer patients regarding the chemotherapy treatment.

Methods: It is a qualitative study, with content analysis based in Bardin. The sample was composed by 100 patients under chemotherapy treatment for colorectal cancer, who were enrolled in A.C. Camargo Cancer Center, São Paulo, Brazil. The data were collected using an interview about the perception of patients concerning chemotherapy. The interviews were audio recorded. For the analysis, we followed three steps: transcription, codification, and answers categorization.

Results: The perceptions of the patients regarding chemotherapy were grouped into four categories: "negative experience", "necessary for disease control", "healing treatment" and "positive experience". The most cited was the negative experience, then, they referred worsening in the emotional level, since they reported sadness, loss of will to perform daily activities, side effects of drugs (hair loss, nausea, diarrhea), and other factors, as well as auxiliary activities necessary to receive treatment.

Conclusions: For patients with colorectal cancer, the chemotherapy treatment causes negative experiences that could contribute for abandoning the treatment. However, they refer to be more strengthened and that they have developed the capability of learning through a painful experience as the chemotherapy. Nurses are responsible for valuing the patient's perception in order to make that his/her experience of illness and treatment possible to be reversed in a positive way, so they can have a better quality of life.
\end{abstract}

Key Words: Chemotherapy, Colorectal cancer, Patient, Perception, Qualitative study

\section{INTRODUCTION}

Cancer is a public health problem that affects more than 32 million people worldwide. ${ }^{1]}$ Colorectal cancer is the third type of cancer with higher incidence. ${ }^{[2]}$ In the United States, the 2016 statistics present colorectal cancer in the third place. ${ }^{[3]}$ In Brazil, the estimates for $2016 / 2017$ predict the occurrence of 34,280 new cases of this type of tumor, placing it in fourth place. ${ }^{[4]}$ The South and Southeast regions of Brazil have the highest number of new cases of colorectal cancer, especially in the states of São Paulo, Rio Grande do Sul, and Rio de Janeiro. Thus, in these cities, this is the third most frequent tumor among men and the second among women, without considering non-melanoma skin tumors. ${ }^{[4]}$

In the experience of the disease, the oncological patient is faced with generating symptoms experiences arising from

\footnotetext{
*Correspondence: Sonia Betzabeth Ticona-Benavente; Email: preciosasonia@gmail.com; Address: Nursing School, Universidade de São Paulo, São Paulo, Brazil.
}

Published by Sciedu Press 
the disease itself, from the treatment and the uncertainty of the results that may interfere in the future life. In this context, different authors refer to the importance of analyzing the emotional sphere of cancer patients, since it has been documented that one-third to one-half of adults with cancer suffer from significant experience of stress. ${ }^{[5,6]}$

In studies about the emotional sphere of cancer patients, the National Comprehensive Cancer Network (NCCN) does not use the term stress in this context because it considers an unpleasant multifactorial emotional experience and psychological (cognitive and behavioral), social and/or spiritual nature that can interfere in the capacity to deal effectively with the disease, its physical symptoms and its treatment. ${ }^{[7]}$

In the current research, the choice of the patient in chemotherapy as the focus of analysis was due to considering this treatment interference in the daily routine of the patients and its greater impact in the physical and emotional sphere and in the experience of the symptoms. In a study of 44 patients with stage IV colorectal cancer receiving chemotherapy, manifestations of toxicity, such as fatigue and insomnia, were identified. ${ }^{[8]}$

Another study conducted in 102 cancer patients identified moderate and severe depression in $10.8 \%$ and $1.9 \%$, respectively, due to the use of antineoplastic therapies ${ }^{[9]}$ and therefore, founded non-adherence to treatment in $48 \%$ of patients. ${ }^{[9]}$ An observational study of 100 patients with advanced colorectal cancer treated with palliative chemotherapy identified symptoms of fatigue, pain, dyspnea, and appetite loss. In addition, the quality of life was deteriorated across time, thus it was found large differences between patients with and without a treatment response for pain (19.0 vs. 37.2 points) and appetite loss (17.4 vs. 32.7 points). ${ }^{[10]}$

Another research conducted with 100 patients with gastrointestinal, breast, lung and ovarian carcinoma evaluated the impact of chemotherapy on its physical or non-physical side effects. Patients indicated the negative impact on the family or spouse, alopecia, fatigue, and negative impact on work or domestic activities, on social and sexual life. ${ }^{[1]}$

Different authors have already explored the perception of cancer patients. However, it remains a necessity, considering the scarcity of studies that address the patient with colorectal cancer. Specially to obtain subsidies that may direct the implementation of effective interventions conducted by the nurse for the management of adverse events and the support offering regarding the experience of the disease and treatment, with better impact on the quality of life.

Therefore, this research aims to explore the perception of patients with colorectal cancer experiencing chemotherapy.

\section{Methodology}

This is a qualitative study that used the content analysis by Bardin's perspective with quantitative approach. ${ }^{[12]}$ This technique makes it possible to analyze the information through systematic procedures and an objective description of the content of the message that allows the inference of knowledge related to the conditions of reception of this message. This approach allows to quantify the frequency of characteristics that are repeated in the content of the participants' answer. ${ }^{[12]}$ Therefore, this method will allow reaching our objective.

The research project was approved by the Research Ethics Committee of the School of Nursing of University of São Paulo (Process 1055/2011) and by the Human Research Ethics Committee of the Antônio Prudente Foundation of the AC Camargo Hospital of São Paulo (Process No 1601/11), São Paulo, Brazil. Still, in consideration of ethical principles, the patients consented to their participation by signing the Free and Informed Consent Term.

Our sample was composed by 100 patients undergoing chemotherapy for colorectal cancer, enrolled in the outpatient chemotherapy unit of the hospital referred above. The participants of this study came from a larger study that evaluated the psychological stress and coping with the experience of the disease. ${ }^{[13]}$ The inclusion criteria were: patients with a medical diagnosis of colon or rectum cancer submitted to outpatient chemotherapy and over 21 years old. The exclusion criteria were: patients with psychiatric disease or any cognitive impairment that made it difficult to participate in the research.

The answer to question in this research "How is it for you to be on chemotherapy?" was obtained through individual interview while the patient received chemotherapy. The interview lasted approximately five minutes, due to the participants of this study caming from a big study that evaluated the psychological stress and coping with the experience of the disease. ${ }^{[13]}$ The responses were recorded in a digital audio recorder. The data collection period took place from December 2011 to February 2012.

For the analysis of the patients' responses, the three steps recommended by Bardin were: Transcription, Encoding, and Categorization. ${ }^{[12]}$ In the first step, we listened and wrote the audio recordings of the patients' answers. In the second step, the data were coded from the identified recording units. In the last step, the categorization was carried out, which consisted in the classification of the elements according to their similarities with the later regrouping, due to common characteristics. For this, it was used descriptive statistics, mainly, absolute and relative frequencies. 


\section{Results}

\subsection{Characterization of the sample studied}

A total of 100 interviews were conducted, 47 women and 53 men, with an average age of 56 years old, most were married $(69 \%)$ and predominantly with higher education (42\%). Regarding the clinical characteristics of the disease, $66 \%$ had colon cancer, with tumor IV staging (50\%), family his- tory of cancer (70\%), no colostomy (72\%), and less than 365 days since diagnosis $($ Median $=175.75$, minimum $=$ 8 and maximum $=1,712$ ), as shown in Table 1 . The most common treatment regimens were FOLFOX (31\%), FLOX (14\%) and 5FU + LEUCOVORIN (9\%) and the most frequent chemotherapy drugs: fluorouracil ( $88 \%)$, leucovorin $(85 \%)$, ansetron $(69 \%)$ and oxaliplatin $(60 \%)$.

Table 1. Statistical characterization of socio-demographic variables of people with colorectal cancer

\begin{tabular}{|c|c|c|c|c|}
\hline & \multicolumn{4}{|c|}{$(n=100)$} \\
\hline & $\mathbf{N}$ & $\%$ & Mean (Sd) & Median (Min-Max) \\
\hline \multicolumn{5}{|l|}{ Sex } \\
\hline Female & 47 & 47.0 & & \\
\hline Male & 53 & 53.0 & & \\
\hline Age (years) & & & $51(13.97)$ & $53(18-85)$ \\
\hline \multicolumn{5}{|l|}{ Marital status } \\
\hline Married & 69 & 69.0 & & \\
\hline Separated/Divorced & 7 & 7.0 & & \\
\hline Single & 15 & 15.0 & & \\
\hline Widowed & 9 & 9.0 & & \\
\hline \multicolumn{5}{|l|}{ Educational level } \\
\hline Illiterate & 1 & 1.0 & & \\
\hline Elementary school (9 years) & 25 & 25.0 & & \\
\hline High school (3 years) & 32 & 32.0 & & \\
\hline Higher education (4 years) & 42 & 42.0 & & \\
\hline \multicolumn{5}{|l|}{ Religious Practices } \\
\hline No & 35 & 35.0 & & \\
\hline Yes & 65 & 65.0 & & \\
\hline \multicolumn{5}{|l|}{ Diagnosis } \\
\hline Colon cancer & 66 & 66.0 & & \\
\hline Rectal cancer & 34 & 34.0 & & \\
\hline Time at diagnosis (days) & & & 365.39 (420.88) & $175.75(8-1,712)$ \\
\hline \multicolumn{5}{|l|}{ Staging (TNM) } \\
\hline II & 13 & 13.0 & & \\
\hline III & 37 & 37.0 & & \\
\hline IV & 50 & 50.0 & & \\
\hline \multicolumn{5}{|l|}{ Type of treatment } \\
\hline Adjuvant & 56 & 56.0 & & \\
\hline Neoadjuvant & 7 & 7.0 & & \\
\hline Palliative & 37 & 37.0 & & \\
\hline \multicolumn{5}{|l|}{ Colostomy } \\
\hline No & 72 & 72.0 & & \\
\hline Permanent & 6 & 6.0 & & \\
\hline Temporary & 22 & 22.0 & & \\
\hline
\end{tabular}

\subsection{Perception of the patients undergoing chemotherapy}

It was possible to group the patients' reports in four broad categories: "negative experience", "necessary for disease control", "healing treatment" and "positive experience" as shown in the following chart (see Figure 1).

Published by Sciedu Press

\subsubsection{Negative experience}

Figure 1 shows the predominance of the category Negative Experience, among these experiences were: anxiety, pain, suffering due to side effects, concern for problems in the supplementary health system and transportation between the home and the hospital, and others. We verify in the reports 
contents the characteristics based on the suffering processes regarding the experience of the chemotherapy treatment with the following declarations: "[...] it is very unpleasant [...] I ask, why to do chemo, why do I have to suffer here [...]." (patient 95). "So, the chemotherapy I think at first for me was easy because I had no reaction, now, actually, if I could not come I would not come, so much that I would give up [sigh]... it's painful” (patient 13). "[...] I cannot miss the chemotherapy, [...] I have been four times (sessions) without chemotherapy, and it was not the doctor's fault, it was by the supplementary health care system [...]" (patient 96).

When we observed a high frequency of patients' perceptions (82\%) about the Negative Experience category, we decided to explore it. Then, it can be observed that most patients perceived chemotherapy as a bad experience due to three factors: emotional, physical and others, as shown in Figure 2.

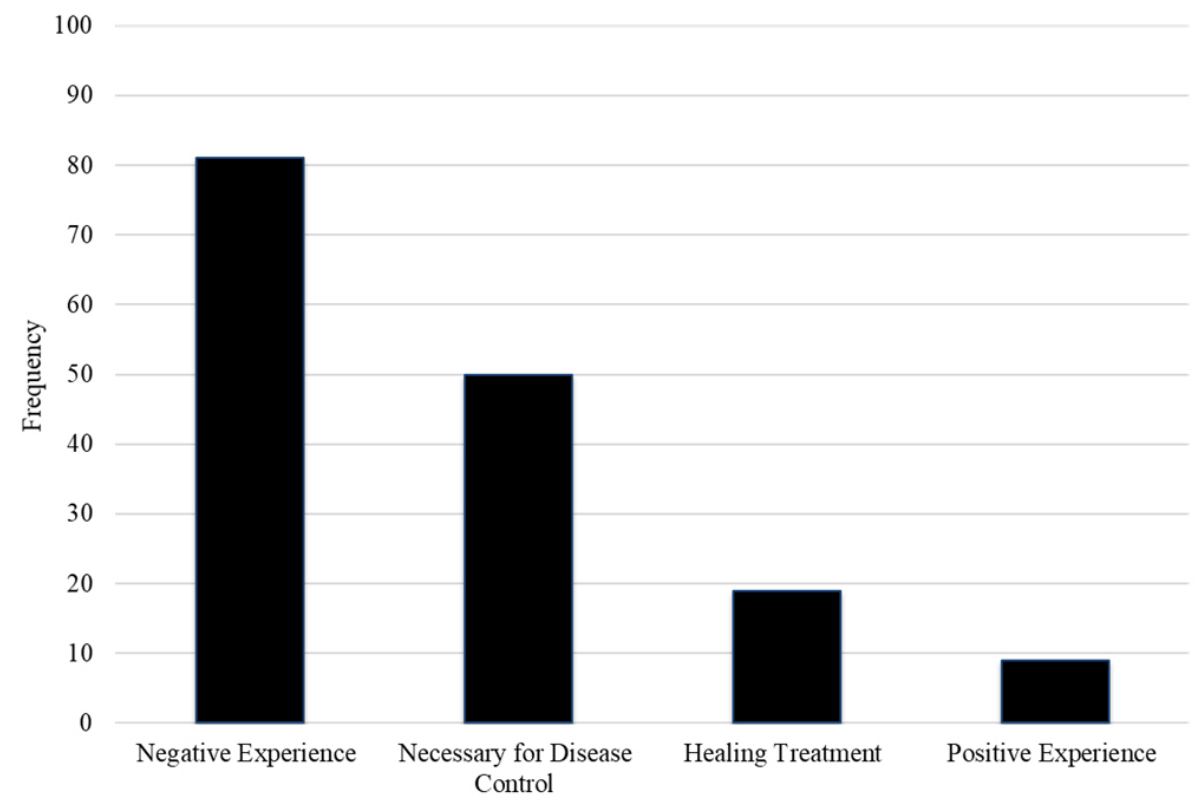

Figure 1. Distribution of patients' perception in four categories

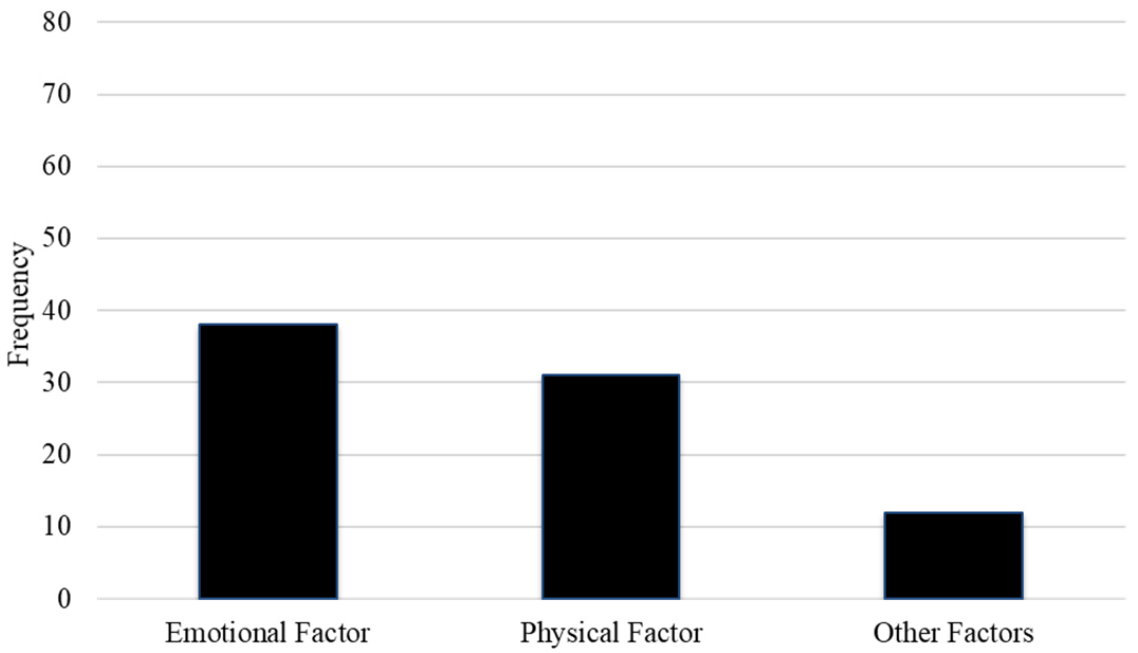

Figure 2. Distribution of patient's perception regarding the negative experiences of the chemotherapy treatment

In the negative experience regarding the emotional factor, the patients express a feeling of revolt and nonconformity due to the non-acceptance of the situation in which they find themselves to be going through this experience that causes suffering. “[...] now I sometimes get rough, half rough because I'm not accepting the situation of becoming a person with cancer [...] I am in [...] could be called an uprising [...]" (patient 64). Thus, for some patients this is a painful 
experience because it causes several changes in the physical and psychological sphere of the human being, " [...] the chemotherapy is painful, for me it is one of the most painful parts of the treatment, it hinders, it messes a lot with the organism, with the whole organism. Psychologically it also shakes" (patient 20) and, even bad to go through and unpleasant "[...] for me it is very bad to be doing (chemotherapy), I do not like to do it [...]." (patient 52). In addition, it is perceived that the chemotherapy is more difficult than other treatments such as surgery, as it is verified in the following statement: "it is one of the most difficult parts that the treatment has [...] even more difficult than the surgery, which I have been through [...]." (patient 20).

In the negative experience, due to the physical factor, they mentioned the frequent and characteristic symptoms of the chemotherapy regimens for colorectal cancer described as discomfort, alopecia, vomitus, and weakness “[...] It's exhausting, right? Because chemo bothers quite badly, whether you like it or not [...] you have discomfort [...] the hair falls [...] the consequences that chemo has [...] it leaves you weak, changes in the body, this part ends up being bad [...]" (patient 26). Still, nausea, inappetence, peripheral nervous disorder, and tiredness were mentioned while having difficulty getting up from bed "[...] there is a lot of nausea [...] I cannot eat, you know [...] it leaves me with the sensitive fingers and a little shock touching cold things [...] to drink too, you know [...] tiredness [...] sometimes I cannot get up [...]" (patient 86).

In negative experience, due to other factors, patients reported a change in routine daily activities due to the time spent in the hospital "[...] time is a complicated factor, I have to quit all my activities to stay here the whole day [...]" (patient 37). In many circumstances, they reported on auxiliary activities needed to receive treatment. Such as waiting for hospital care, due to the delay in the release of the treatment by the supplementary healthcare system "[...] I get a little annoyed when I get here and I'll do the exams and I am said: wait a little longer whether I will or not be able to do it [...]" (patient 28). Thus, difficulties in the home-hospital transportation, especially when the patient lives in another city, a phenomenon that impacts on the exercise of work "[...] the displacement from Manaus to São Paulo brings me several inconveniences and disorders to work [...] chemotherapy makes it difficult for me to have a normal life [...]." (patient 44 ). Still, they refer to the impossibility of carrying out ordinary activities such as sunbathing "[...] for me it disturbs my life, [...]I cannot travel far, sunbathe [...]” (patient 31).

\subsubsection{Treatment need for disease control}

Regarding the category of Treatment Need for Disease Control, when patients have spoken with the doctor, they are aware of the condition in which they are and are aware of the intention to control the disease and the feeling of resignation. So, it is necessary to receive the chemotherapy to prevent the tumor growth in patients at the baseline, or for palliative purposes in patients with advanced stages. Thus, the following statements stand out: "[...] it is necessity for me to do chemo [...]." (patient 25). "[...] since I'm sick, I have to do it, if I was good, I was not doing it [...].” (patient 16). "[...] my case has no cure, I'm going to do chemotherapy, so I do not have pain [...] it's for the rest of my life." (patient 18)

\subsubsection{Healing treatment}

In the Healing Treatment category, patients have hope of healing through treatment; although in medicine it does not use the term "cure" but rather of survival because of the possibility of recurrence of the tumor. It is still perceived that they are informed about the duration of the treatment, and have the feeling of acceptance. "[..] I am aware that I need to go through this (chemotherapy), you get it, I think so,[...], what I need is to think about my healing, so if I have to go through it, I'm gonna take it easy, you get it, I think so" (patient 80). "For me it's normal, it's something that must be done, I'll do it normally, depending on what I must do [...] I'll do it for six months or so [...] I'll do it quietly, without any problem" (patient 30). "For me [...] it is a cure treatment [...]" (patient 43)

\subsubsection{Positive experience}

Among the statements that referred to chemotherapy as a Positive Experience, they pointed out that the treatment was a blessing from God; it was also seen as an opportunity for family strengthening, value restructuring, and learning from that experience: "[...] for me, it is a blessing of God to be doing chemo [...]" (patient 4). "[...] sometimes I think it could end soon, but it has also been a moment of more union in the family [...]" (patient 32). "[...] it is a life time experience, [...] my values have changed [...] I am taking it as a teaching, as a learning [...]" (patient 62)

\section{Discussion}

This study reveals the experience of colorectal cancer patients on chemotherapy according their perception, defined as: "negative experience", "necessary for disease control", "healing treatment" and "positive experience".

The emotional factor, side effects, and other factors, such as the auxiliary activities needed to receive treatment have been set as a negative experience associated with chemotherapy. Each of these factors will be discussed below. 
Patients do not accept the fact that they are ill and must undergo chemotherapy, which causes revulsion and nonconformity to diagnosis and treatment. Another research has identified the presence of negative feelings, such as: fear, concern, hopelessness, anger, shock, injustice, and denial, as well as insomnia, weakness and inappetence; feelings experienced when they experienced changes in their daily routine to deal with the symptoms, diagnosis, and treatments. They are related to the diagnosis because it still carries the death sentence stigma while the chemotherapy is associated with the side effects. ${ }^{[14]}$ In this sense, it is perceived that this feeling is part of the process of coping with the disease and remains while the patient does not accept its clinical condition.

In addition, it is verified that despite the medical-scientific advances in chemotherapy drugs for colorectal cancer, it has made them more active and with reduced toxicity ${ }^{[15]}$ to reduce the discomfort among patients; there are still significant physical manifestations that trigger different signs and symptoms that may hinder the performance of daily activities. ${ }^{[16,17]}$ Manifestations that make chemotherapy a negative experience.

Furthermore, in this study, most patients received fluorouracil, leucovorin, ansetron and oxaliplatin. Drugs that commonly cause nausea, vomiting, diarrhea, peripheral neuropathy, fever, and neutropenia. ${ }^{[18-20]}$ In this sense, a longitudinal study conducted in 10 patients with colorectal cancer evaluated the effects of oxaliplatin neurotoxicity; 3, 6 and 12 months after the treatment, patients reported feeling lack of energy, pain, discomfort with cold, and difficulty touching objects. These effects have made them sad because they affected their daily lives. ${ }^{[16]}$

Another study conducted in 44 patients with colorectal cancer in stage IV receiving the drug-based chemotherapy treatment: Irinotecan, 5-Fluorouracil, and Leucovorin, evaluated the quality of life in three moments (in the first, third and fourth cycles of the treatment). Thus, in the first cycle it was identified some sexual problems; in the third cycle, fatigue, sleeping problems and sexual functioning; in the fourth cycle, sleeping problems, sexual problems, weight loss, and appetite disappeared. ${ }^{[8]}$ Thus, it is perceived that the sexual aspect was the change quoted at all the three moments.

In another research conducted in 21 breast cancer patients undergoing chemotherapy, the patients reported that alopecia, a side effect of chemotherapy, was the most traumatic and painful experience. In addition, they reported that they would not have undergone treatment if they knew of the suffering that the treatment would cause. ${ }^{[21]}$ Another study carried out on 100 oncology patients, the most common effects were: alopecia, fatigue, negative impact on the family or spouse, work or domestic activities, as well as effects on the social and sexual life. ${ }^{[11]}$ This induces us to reflect on side effects with negative impact on the patient's quality of life.

Still, in this research, the auxiliary activities necessary to receive the treatment generate discomfort, since these activities must be incorporated as part of the daily routine of the patient. In this sense, a study carried out in Australia analyzed the patients' perception on chemotherapy, and identified that the patients reported greater discomfort due to the prolonged time of each treatment session and the limitations to perform daily activities. ${ }^{[2]}$ On the contrary, in our research, patients from other regions had to make long journeys to receive the treatment due to scarce centers specialized in oncology. Also, there are reports about the administrative difficulty regarding the supplementary health service, difficulty that exceeds the patient control, and deserves hospital management attention. Thus, it is perceived that not only the factors intrinsic to the treatment cause concern and discomfort, but there are also other factors that contribute to the patient's discomfort.

In addition, when the patient indicates that the chemotherapy is needed to control the disease, he/she appears to be aware of his or her condition as a patient and understands that chemotherapy will help him or her prolong life by controlling the tumor growth or relieve the pain, a common symptom among cancer patients. This is a cognitive process by which patients can cope with the situation in which they find themselves. Thus, a qualitative study conducted in seven cancer patients explored the process of coping with the disease process based on the data-based theory, and identified that patients after understanding that the diagnosis of cancer cannot be altered, accepted to follow all the treatment, be it surgical, radiotherapy and/or chemotherapy. ${ }^{[14]}$

On the contrary, when the patient perceives that chemotherapy is a healing treatment, it seems to express the possibility of a cure that the patient has, once the tumor is diagnosed at the initial stage, ${ }^{[23]}$ or his/her willingness to cope with the disease in order to return to life without it. Thus, a survey conducted in Switzerland with 10 colorectal cancer patients in stages III and IV, who had completed chemotherapy, revealed that the patient has the desire to be "cured" as the main aim. Although the side effects remained after treatment, they expected them to disappear, believing in healing and hoping to be healthy again and have a "normal life". ${ }^{[16]}$ However, this desire for cure is also found in patients in advanced stages, as a study conducted with 1,193 patients in palliative chemotherapy for stage IV colorectal and lung cancer expressed the expectation of cure of the disease despite their clinical condition. ${ }^{[2]}$ This gives us indications that the pa- 
tient embraces the feeling of hope despite the circumstances, a feeling of the human being.

On contrary, the perception of patients who define this experience as positive, despite the negative effects related to the chemotherapeutic treatment, stands out for the positive aspect of the situation, valuing the spirituality, the reason for learning, and the opportunity for strengthening the family unity. Another research has found similar results, by instance, a qualitative research, together with patients with breast cancer, endometrial and leukemia, indicates that when patients reevaluate the meaning of life, they alter their old value systems, a phenomenon that allows them to live the rest of their lives from a more positive perspective. ${ }^{[14]}$ Another study carried out in patients with breast cancer states that the positive attitude of the patients, along with the will to live, favors the acceptance of the disease and adherence to the treatment. ${ }^{[25]}$ Although the studies presented were carried out in patients with breast cancer, it can be seen from our results that colorectal cancer patients also have this proactive coping with the disease.

\section{Conclusion}

The present study explored the patients' perspective regarding the experience of outpatient chemotherapy. Our results provide evidence of the need for individualized patient care and appropriate support throughout the chemotherapy treatment, considering that chemotherapy may be seen as a difficult phase of the disease process.

It is, therefore, nurses' responsibility to become aware of the importance of their actions, according the patients' experience, specifically on the emotional, physical and hospital care aspects, identified as negative experiences in this research. In addition, it is suggested to increase the knowledge about patients' positive experience and to discover the psycho-emotional characteristics of these patients.

We encourage the identification of the patients' experience to provide appropriate support to these patients and in contribution with the integrality of the care offered. Also, this research serves as a basis for future research with patients with other types of neoplasms and with other designs; and it provides necessary subsidies for the construction of intervention programs aimed at promoting and development of coping strategies that assist patients in chemotherapy treatment, to contribute consequently to a better quality for them.

\section{CONFLicts of InTEREST Disclosure}

All authors declare no conflicts of interest. This research does not have a financial relationship with any organization. We have full control of all the primary data and we agree to allow the journal to review it if requested.

\section{REFERENCES}

[1] WHO. GLOBOCAN 2012: Estimated cancer incidence, mortality and prevalence worldwide in 2012 In: Cancer IAfRo, editor.: World Health Organization; 2012.

[2] WHO. Estimated Cancer Incidence, All ages: Both sexes. In: GLOBOCAN, editor.: International Agency for Research on Cancer. 2012.

[3] ACS. Colorectal Cancer Facts \& Figures 2014-2016. Atlanta: American Cancer Society; 2014.

[4] INCA. Estimativa 2016 Incidência de Câncer no Brasil. 2016.

[5] Carlson L, Bultz B. Benefits of psychosocial oncology care Improved quality of life and medical cost offset. Health and Quality of Life Outcomes. 2003. 1-8 p. http://doi .org/10.1186/1477-7525-1-8

[6] Hofman M, Morrow G, Roscoe J, et al. Cancer patients expectations of experiencing treatment related side effects. Cancer. 2004; 101(4): 851-7. http://doi.org/10.1002/cncr. 20423

[7] Holland JC, Andersen B, Breitbart WS, et al. Distress management. NCCN Guidelines: National Comprehensive Cancer Network. 2011. 190-209 p. http://doi.org/10.6004/jnccn. 2013.0027

[8] Arraras J, Vera R, Martínez M, et al. Quality of life assessment through the EORTC questionnaires of colorectal cancer patients in advanced disease stages. Clinical and Translational Oncology. 2006; 8(9). http://doi.org/10.1007/s12094-006-0036-6

[9] de Souza B, Pires F, Dewulf N, et al. Patients on chemotherapy: Depression and adherence to treatment. Revista da Escola de Enfer- magem. 2013; 47(1): 61-8. http://doi .org/10.1590/S0080-6 2342013000100008

[10] Mayrbaurl B, Giesinger JM, Burgstaller S, et al. Quality of life across chemotherapy lines in patients with advanced colorectal cancer: A prospective single-center observational study. Support Care Cancer. 2016; 24(2): 667-74.

[11] Carelle N, Piotto E, Bellanger A, et al. Changing patient perceptions of the side effects of cancer chemotherapy. Cancer. 2002; 95(1): 155-63.

[12] Bardin L. Análise de conteúdo. Lisboa: Edições 70; 2012.

[13] Ticona S, Santos E, Siqueira A. Gender differences in perceived stress and coping strategies among colorectal cancer patients receiving chemotherapy. Aquichan. 2015; 15(1): 9-20. http://doi .org/ 10.5294/aqui.2015.15.1.2

[14] Chen PY, Chang HC. The coping process of patients with cancer. Eur J Oncol Nurs. 2012; 16(1): 10-6.

[15] Braun M, Seymour M. Balancing the efficacy and toxicity of chemotherapy in colorectal cancer. Therapeutic Advances in Medical Oncology. 2011; 3(1): 43-52. http://dx.doi.org/10.1177/175 8834010388342

[16] Drott J, Starkhammar H, Kjellgren K, et al. Supportvie Care Cancer 2016; 24: 3455-61. http://dx.doi.org/10.1007/s00520-016 $-3179-1$

[17] Tofthagen C, McAllister D, McMillan S. Peripheral neoruopathy in patients colorectal cancer receiving oxaliplatin. Clin J Oncol Nurs. 
2011; 15(2): 182-8. http://doi.org/10.1188/11.CJON.182-1 8

[18] Kumar A, Peixoto R, Kennecke H, et al. Effect of adjuvant FOLFOX chemotherapy duration on outcomes of patients with stage III colon cancer. Clinical Colorectal Cancer and Other Gastrointestinal Malignancies. 2015; 14(4): 262-8. http://doi .org/10.1016/j.clcc .2015 .05 .010

[19] Enzinger PC, Burtness BA, Niedzwiecki D, et al. CALGB 80403 (Alliance)/E1206: A Randomized Phase II Study of Three Chemotherapy Regimens Plus Cetuximab in Metastatic Esophageal and Gastroesophageal Junction Cancers. J Clin Oncol. 2016; 34(23): 2736-42. PMid:27382098 https://doi.org/10.1200/JC0.2015.65.50 92

[20] Sharif S, O'Connell MJ, Yothers G, et al. FOLFOX and FLOX regimens for the adjuvant treatment of resected stage II and III colon cancer. Cancer Invest. 2008; 26(9): 956-63. PMid:18798075 https://doi.org/10.1080/07357900802132550

[21] Kim IR, Cho JH, Choi EK, et al. Perception, Attitudes, Preparedness and Experience of Chemotherapy-Induced Alopecia among Breast
Cancer Patients: a Qualitative Study. Asian Pacific Journal of Cancer Prevention. 2012; 13(4): 1383-8. http://doi.org/10.7314/ap jcp. 2012.13.4.1383

[22] Coates A, Anbraham S, Kaye S, et al. On the receiving end-Patient perception of the side-effects of cancer chemotherapy. Eur J Cancer Clin Oncol. 1983; 19(2). https://doi.org/10.1016/0277-537 9 (83) $90418-2$

[23] OPS. La OPS/OMS insta a implementar medidas accesibles y costoeficaces para evitar que las personas mueran por cáncer antes de tiempo. Organización Panamericana de la Salud; 2015.

[24] Weeks JC, Catalano PJ, Cronin A, et al. Patients' expectations about effects of chemotherapy for advanced cancer. N Engl J Med. 2012; 367(17): 1616-25. PMid:23094723 https://doi.org/10.1056/ NEJMoa1204410

[25] Leite F, Amorim M, de Castro D, et al. Coping strategies and the relationship with sociodemographic conditions of women with breast cancer. Acta Paulista de Enfermagem. 2012; 25(2): 211-7. http://doi.org/10.1590/S0103-21002012000200009 